\section{Sinnvolle Kombinationen bei Bluthochdruck \\ Vorteile bei Morbidität und Mortalität}

- Bei der antihypertensiven Kombinationstherapie sollten auch pharmakokinetische Gesichtspunkte wie unterschiedlich lange Plasmahalbwertszeiten ins therapeutische Kalkül mit einbezogen werden, betonte Prof. Thomas Mengden, Bad Nauheim. Der Einsatz des beliebten, jedoch nur kurz wirksamen Diuretikums Hydrochlorothiazid (HCT) wirft vor diesem Hintergrund Fragen auf.

Das britische NICE-(National Institute for Health and Clinical Excellence-)Institut hat eine dieser Fragen inzwischen dezidiert beantwortet. Immer dann, wenn essentielle Hypertoniker auf ein Diuretikum ein- oder umgestellt werden sollen, sollten Substanzen wie Chlortalidon oder Indapamid der Vorzug etwa gegenüber dem nur kurz wirksamen HCT gegeben werden. Die vom NICE favorisierten Diuretika zeichnen sich im Vergleich mit HCT durch eine deutlich längere Halbwertszeit aus, machte Prof. Heribert Schunkert, Lübeck, deutlich. Indapamid hat darüber hinaus eine er- höhte NOS-(Stickstoffmonoxyd-Synthase-) Aktivität sowie eine erhöhte endotheliale NOS-Expression.

\section{Erfolgreiche Umstellung}

Die genannten Faktoren könnten erklären, warum sich in der von Dr. Walter Sehnert, Dortmund, vorgestellten Pilot-Studie die Umstellung von Ramipril/HCT auf die Fixkombination Perindopril/Indapamid (BiPreterax ${ }^{\circledast}$ N) bei Typ-2-Diabetikern mit unzureichend eingestelltem Blutdrucks offenbar bezahlt gemacht hat. Acht Wochen nach der Umstellung war es $u$. a. zu einer signifikanten Absenkung der systolischen und diastolischen Langzeitblutdruck-Werte sowie auch einer deutlichen Absenkung des zentralen Blutdrucks gekommen.

Gute Argumente für die (Fix-)Kombination von Perindopril und Indapamid ergeben sich nach den Worten von Prof. Stephan Jacob, Villingen-Schwenningen, aus den Ergebnissen der ADVANCE-Studie. Zusätzlich zu einer leitliniengerechten Blutdruck-

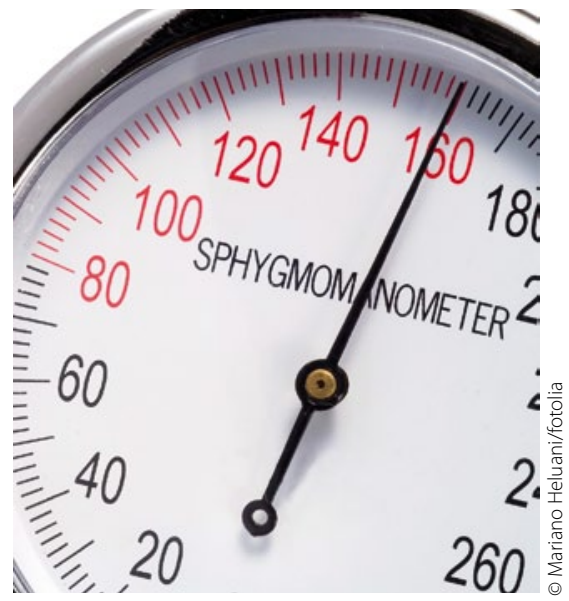

Die Plasmahalbwertszeit von Diuretika mit ins therapeutische Kalkül einbeziehen!

therapie gegeben, ergaben sich im Vergleich zur Kontrollgruppe für die genannte Kombination Vorteile im Hinblick auf die kardiovaskuläre Mortalität und Morbidität. Insbesondere ließ sich auch das Fortschreiten einer Nephropathie substanziell senken.

- Ludger Riem

Quelle: Symposium „Effektive Substanzen sinnvoll kombinieren - der Schlüssel zum Erfolg?", Kongress der Deutschen Hochdruckliga e.V., Köln, November 2011 (Veranstalter: Servier)

\title{
Therapie des Typ-2-Diabetes
}

\section{Ältere Diabetiker sind eine besondere Herausforderung}

— Bei älteren Typ-2-Diabetikern besteht meist eine Komorbidität. Neben kardialen Erkrankungen und Depressionen kommen die chronische Niereninsuffizienz häufig vor, bei der eine Reihe oraler Antidiabetika wie Sulfonylharnstoffe und Metformin problematisch, oft sogar kontraindiziert sind, sagte Prof. Matthias Blüher, Leipzig. Gerade hier seien Gliptine wie Vildagliptin (Galvus ${ }^{\circledR}$ ) eine wertvolle Bereicherung.

In einer klinischen Studie wurde die Wirksamkeit und Sicherheit von Vildagliptin bei 31 Typ-2-Diabetikern über 75 Jahre untersucht. Nach 24-wöchiger Behandlung mit Vildagliptin + Metformin sank der $\mathrm{HbA}_{1 c}$-Wert um $1,1 \%$, ohne dass Hypoglykämien auftraten. „Überraschenderweise war Vildagliptin bei diesen älteren Patienten sogar effektiver als bei jüngeren", so Blüher.
Anhand von Daten aus dem Versorgungsalltag von 1645 Typ-2-Diabetikern konnte gezeigt werden, dass Vildagliptin eine signifikant stärkere Senkung des $\mathrm{HbA}_{1 \mathrm{c}}$-Wertes bewirkt als andere DPP4-Inhibitoren. Unter Vildagliptin sank der $\mathrm{HbA}_{1 c}$-Wert um 1,1\%, bei anderen DPP4Inhibitoren im Durchschnitt um 0,9\%. Auch in der PROVIL-Studie konnte durch die Kombination Vildagliptin + Metformin (Eucreas ${ }^{\circledR}$ ) der $\mathrm{HbA}_{1 c^{-}}$Wert um ca. 0,3\% stärker gesenkt werden als mit anderen oralen Zweifachkombinationen.

\section{Auch bei Niereninsuffizienz sicher}

In einer placebokontrollierten doppelblinden Multicenterstudie wurde die Sicherheit und Verträglichkeit von $50 \mathrm{mg}$ Vildagliptin bei 294 Patienten mit moderater und bei 221 Patienten mit schwerer chronischer Niereninsuffizienz untersucht. „Im Vergleich zur Placebogruppe traten in der Vildagliptin-Gruppe unerwünschte $\mathrm{Er}$ eignisse nicht vermehrt auf", so Blüher. Der $\mathrm{HbA}_{1 \mathrm{c}}$-Wert konnte bei moderater chronischer Niereninsuffizienz um 0,7\% (ausgehend von 7,9\%) und bei schwerer chronischer Niereninsuffizienz sogar um 0,9\% gegenüber einem Ausgangswert von 7,7\% gesenkt werden. „Angesichts dieser positiven Studiendaten hat die Europäische Zulassungsbehörde eine positive Beurteilung für Vildagliptin für die Behandlung von Typ2-Diabetikern mit mäßiger oder schwerer Nierenschädigung gegeben", so Blüher.

- Dr. med. Peter Stiefelhagen

Quelle: Meet-the-Expert "Nutzen von Vildagliptin bei älteren Patienten und Patienten mit Niereninsuffizienz", DDG-Herbsttagung, Berlin, November 2011 (Veranstalter: Novartis) 